\title{
The cosmic web and microwave background fossilize the first turbulent combustion
}

\author{
Carl H. Gibson ${ }^{1}$ and R. Norris Keeler ${ }^{2}$ \\ ${ }^{1}$ MAE and SIO, Univ. of Cal. San Diego, \\ La Jolla, CA, 92093-0411, USA \\ email: cgibson@ucsd.edu \\ ${ }^{2} 6652$ Hampton Park Court, \\ McLean VA 22101, USA \\ email: rnkeeler@verizon.net
}

\begin{abstract}
Collisional fluid mechanics theory predicts a turbulent hot big bang at Planck conditions from large, negative, turbulence stresses below the Fortov-Kerr limit $\left(<-10^{113} \mathrm{~Pa}\right)$. Big bang turbulence fossilized when quarks formed, extracting the mass energy of the universe by extreme negative viscous stresses of inflation, expanding to length scales larger than the horizon scale ct. Viscous-gravitational structure formation by fragmentation was triggered at big bang fossil vorticity turbulence vortex lines during the plasma epoch, as observed by the Planck space telescope. A cosmic web of protogalaxies, protogalaxyclusters, and protogalaxysuperclusters that formed in turbulent boundary layers of the spinning voids are hereby identified as expanding turbulence fossils that falsify $\Lambda C D M H C$ cosmology.
\end{abstract}

Keywords. turbulence, cosmology, dark matter, dark energy.

\section{Introduction}

Yakov Borisovich Zeldovich pioneered the difficult field of turbulent combustion, and would have appreciated the complexities that arise when collisional fluid mechanics, general relativity, self-gravitational-stratification, fossil turbulence, fossil turbulence waves, and beamed zombie turbulence maser action mixing chimneys (journalofcosmology.com volume 21) are combined in the first turbulent combustion of the big bang (JoC volumes 15-24), Gibson (2004), Gibson (2005), Keeler and Gibson (2012).

Space telescopes show distinctive fossil turbulence patterns in the cosmic web and in the cosmic microwave background that confirm a big bang turbulent combustion mechanism, where turbulence is defined by inertial vortex forces and negative Fortov-Kerr pressures [Fortov (2012)] extract mass-energy from turbulence needed to trigger inflation by gluon viscous stresses of the strong force freeze-out. Such turbulence always cascades from small scales to large (see journalofcosmology.com volumes 15-23) and leaves patterns termed fossil turbulence in a variety of hydrophysical fields that preserve information about the previous turbulence. For astrophysical sample calculations and physical constants, see Gibson (2000). Photon viscosity during the plasma epoch explains the fragmentation of protogalaxies, and the observations showing all galaxies have the same mass, Tyson, J. A. \& P. Fischer (1995).

The cosmic microwave background spectrum reveals fossil turbulence patterns at large wavelengths (now $>10^{25} \mathrm{~m}$ ) fossilizing big bang turbulent combustion, and smaller wavelengths fossilizing viscous gravitational fragmentation of the plasma epoch at $10^{12}$ seconds to produce $\approx 10^{24} \mathrm{~m}$ Superclusters and Superclustervoids in the turbulent boundary layers of spinning SuperSuperclustervoids, now $\approx 2 \times 10^{25} \mathrm{~m}$ in diameter. See JoC vol. 24 
for details. Protogalaxies formed at $10^{12} \mathrm{~s}$ serve as fluid particles for the weak boundary layer turbulence.

The CMB spectral turbulence pattern is a single peak reflecting a highly concentrated vortex, and two secondary peaks reflecting transverse secondary vortices at right angles that stretch the primary vortex by inertial vortex forces into a tubular shape about one part per million of the Kolmogorov space time at the Planck conditions of the big bang. Fortov-Kerr negative stresses less than $-10^{113} \mathrm{~Pa}$ extract mass-energy from the vacuum.

The plasma turbulence peak and its two harmonics has been misinterpreted as sonic oscillations of trapped baryons in cold dark matter potential wells. $\Lambda C D M H C$ cosmology is generally falsified by the fluid mechanically based cosmology and observations presented, where cold dark matter, dark energy and hierarchical clustering of CDM clumps is questioned.

Schild (1996) proved the dark matter of galaxies is Earth-mass planets of hydrogenhelium, confirming fluid mechanical predictions of Gibson (1996) that plasma superclusters and clusters of protogalaxies fragmented into Jeans mass clumps of primordial planets at the plasma to gas transition at time $10^{13}$ seconds. Stars and larger planets are formed in these proto-globular-star-cluster clumps by binary mergers. Since $\approx 10^{80}$ dark matter planets are produced by the big bang, numerous habitats for the formation of life are provided by HGD cosmology, Gibson, Schild, \& Wickramasinghe (2011).

\section{Discussion}

Turbulence and gravitational structure formation are absolutely unstable random processes. Several critical length scales (Schwarz scales) distinguish different structure formation regimes and hydrogravitational phase diagrams, Gibson (1996). The most important length scale for cosmological structure formation is the viscous-gravitational Schwarz scale $L_{S V} \approx[\gamma \nu / \rho G]^{1 / 2}$. The rate-of-strain $\gamma$ is $\approx 1 / \mathrm{t}$. The kinematic viscosity $\nu$ during the plasma epoch is huge (the photon viscosity), so the fragmention mass $\left(10^{44} \mathrm{~kg}\right)$ is that of a galaxy, Gibson (2000). After transition to gas at $10^{13} \mathrm{~s}, \nu$ decreases by a factor of $10^{13}$, giving a planetary $\left(10^{24} \mathrm{~kg}\right)$ fragmentation mass. This matches that observed by quasar microlensing of a foreground galaxy, Schild (1996).

\section{Conclusions}

Evidence of empty SuperSuperVoids supports the hypothesis of big bang turbulence. The cosmic web is identified as linear structures characteristic of fossil turbulent vortex lines in the turbulent boundary layers of these spinning big bang fossil turbulence vortex lines that have triggered structure formation during the plasma epoch.

\section{References}

Fortov, V. E. 2012, J. of Cosmology, 18, 14, 8105-8138.

Gibson, C. H. 1996, Appl. Mech. Rev., 49, 299

Gibson, C. H. 2000, J. Fluids Eng., 122, 830-835. JoC, 23, 11, 11206-11212.

\&Gibson, C. H., R. E. Schild, \& N. C. Wickramasinghe 2011, Int. J. of Astrobiology, 10 (2): 83-98. The origin of life from primordial planets.

Gibson, C. H. 2004, Flow Turbul. Combust., 72, 161-179.

Gibson, C. H. 2005, Combust. Sci. and Tech., 177, 1049-1071. arXiv:astro-ph/0501416

Keeler, R. N. \& C. H. Gibson 2012, J. of Cosmology, 18, 13, 8095-8104.

Schild, R. E. 1996, ApJ, 464, 125.

Tyson, J. A. \& P. Fischer 1995, ApJ, 446, L55. 